\title{
BOUNDARY LAYER FORMATION IN THE TRANSITION FROM THE POROUS MEDIA EQUATION TO A HELE-SHAW FLOW
}

\author{
FORMATION DE COUCHE LIMITE LORS DE LA \\ TRANSITION ENTRE L'ÉQUATION DES MILIEUX \\ POREUX ET LE PROBLÈME DE HELE-SHAW
}

\author{
O. GIL ${ }^{\mathrm{a}}$, F. QUIRÓS ${ }^{\mathrm{b}, *}$ \\ a Instituto de Matemática y Estadística "Prof. Ing. Rafael Laguardia", Facultad de Ingeniería, \\ Universidad de la República Oriental del Uruguay, Casilla de Correo 30, Montevideo, Uruguay \\ b Departamento de Matemáticas, Universidad Autónoma de Madrid, 28049 Madrid, Spain
}

Received 7 June 2000, revised 8 May 2002

Abstract. - Let $u_{m}(x, t)$ be the solution to the Porous Media Equation, $u_{t}=\Delta u^{m}$, in a domain $\Omega \subset \mathbb{R}^{n}$, with initial data $u_{m}(x, 0)=f(x)$ and boundary data $u_{m}^{m}(x, t)=g(x)$. Let $v_{m} \equiv u_{m}^{m}$. We prove the convergence as $m$ goes to infinity of the pair $\left(u_{m}, v_{m}\right)$ to a pair $\left(u_{\infty}, v_{\infty}\right)$ which is a weak solution of the Hele-Shaw problem with boundary data $v_{\infty}=g$ and initial data $u_{\infty}(x, 0)=\tilde{f}(x)$, where $\tilde{f}(x)$ is the projection of the initial data $f(x)$ into a 'mesa'. We also prove the convergence of the positivity sets of the functions $u_{m}$ to the positivity set of $u_{\infty}$. For large but finite $m$ a boundary layer connecting the initial data $f(x)$ and its projection $\tilde{f}(x)$ appears. We analyze the convergence of solutions and positivity sets in this boundary layer by introducing a suitable time scale. All our results hold true also for the Cauchy problem $\left(\Omega=\mathbb{R}^{n}\right.$, no boundary data).

(C) 2003 L'Association Publications de l'Institut Henri Poincaré. Published by Elsevier B.V. All rights reserved

MSC: 35K65; 35R35; 76S05

Keywords: Hele-Shaw problem; Porous media equation; Mesa problem; Boundary layer; Singular limit; Free boundary

RÉSUMÉ. - Soit $u_{m}(x, t)$ la solution de l'équation des milieux poreux $u_{t}=\Delta u^{m}$, dans un domaine $\Omega \subset \mathbb{R}^{n}$, avec donnée initiale $u_{m}(x, 0)=f(x)$ et $u_{m}^{m}(x, t)=g(x)$ fixée sur la frontière de $\Omega$. Soit $v_{m} \equiv u_{m}^{m}$. Nous montrons la convergence, lorsque l'exposant $m$ va à l'infini, de la paire $\left(u_{m}, v_{m}\right)$ vers $\left(u_{\infty}, v_{\infty}\right)$, qui est l'unique solution faible du problème de Hele-Shaw avec donnée

\footnotetext{
* Corresponding author.

E-mail addresses: omargil@ fing.edu.uy (O. Gil), fernando.quiros@uam.es (F. Quirós).
} 
$v_{\infty}=g$ au bord de $\Omega$, et donnée initiale $u_{\infty}(x, 0)=\tilde{f}(x), \tilde{f}(x)$ étant la projection de $f(x)$ sur une 'mesa'. Nous prouvons aussi la convergence des ensembles de positivité des functions $u_{m}$ vers celui de $u_{\infty}$. Pour $m$ grand, mais fini, une couche limite apparaît, faisant la connexion entre la donnée initiale $f(x)$ et sa projection $\tilde{f}(x)$. Nous étudions la convergence des solutions et des ensembles de positivité dans cette couche limite, introduisant une échelle de temps convenable. Les résultats de ce papier contiennent également le cas du problème de Cauchy pour l'équation des milieux poreux $\left(\Omega=\mathbb{R}^{n}\right.$, aucune donnée n'est fixée sur la frontière de $\Omega$ ).

(C) 2003 L'Association Publications de l'Institut Henri Poincaré. Published by Elsevier B.V. All rights reserved

\section{Introduction and results}

We consider the behaviour of non-negative solutions $u_{m}(x, t)$ to the Porous Media Equation (PME for short)

$$
u_{t}=\Delta u^{m}, \quad(x, t) \in \Omega \times \mathbb{R}^{+},
$$

as $m$ goes to infinity. The spatial domain $\Omega$ is an open subset of $\mathbb{R}^{n}$. We prescribe initial data

$$
u(x, 0)=f(x) \geqslant 0, \quad x \in \Omega,
$$

independent of $m$, where $f$ is measurable, bounded and compactly supported. If the boundary $\partial \Omega$ of $\Omega$ is non-empty, we assume that it is a smooth and bounded hypersurface in $\mathbb{R}^{n}$ and prescribe boundary data

$$
u^{m}(x, t)=g(x) \geqslant 0, \quad(x, t) \in \partial \Omega \times \mathbb{R}^{+},
$$

which will also be taken independent of $m$. We will take $g$ as the trace of a function in $W^{1,2}(\Omega) \cap L^{\infty}(\Omega)$. Observe that we are not asking $\Omega$ to be bounded. Thus, we cover both the case of bounded domains and of exterior domains.

For $m>1$ the PME is a degenerate equation having the finite speed of propagation property, that is, if $f$ is compactly supported, the same is true for $u_{m}(\cdot, t)$ for all $t>0$, and the positivity sets $\left\{u_{m}(\cdot, t)>0\right\}$ are bounded for all $t>0$.

The aim of this paper is twofold: (i) To study the limit $m \rightarrow \infty$ for nontrivial boundary data, $g \not \equiv 0$, with inconsistent (see below) initial data, $\|f\|_{\infty}>1$, an interesting case not previously considered in the literature, and (ii) To study the behaviour as $m \rightarrow \infty$ of the posivity sets $\left\{u_{m}(\cdot, t)>0\right\}$. The convergence of the positivity sets as $m \rightarrow \infty$ has not been studied before, not even for the Cauchy problem, a case which is also covered here.

Eq. (1.1) with $m>1$ is used to describe the infiltration of fluids in porous media. In this model the function $u$ stands for the density of the fluid. Thus, the positivity set $\left\{u_{m}(\cdot, t)>0\right\}$ is the region occupied by the fluid at time $t$. The evolution of the flow is controlled by the pressure,

$$
p=\frac{m}{m-1} u^{m-1} .
$$

Indeed, the velocity of the fluid $\vec{v}$ satisfies Darcy's law

$$
\vec{v}=-\nabla p,
$$


and the positivity sets $\left\{u_{m}(\cdot, t)>0\right\}$ evolve accordingly. It is easy to check that the pressure satisfies

$$
p_{t}=(m-1) p \Delta p+|\nabla p|^{2} .
$$

Formally Darcy's law is valid in the limit $m \rightarrow \infty$. Hence we expect that the pressure $p$ will play an important role when describing the behaviour of solutions and positivity sets for large values of $m$. Observe that for such large values we have $p \sim u^{m}$. Thus, (1.3) basically means that we are imposing the pressure at the boundary, something that seems rather sensible from the physical point of view.

Keeping the above remarks in mind, we introduce the generalized pressure

$$
v=u^{m} .
$$

This will simplify the formulas, while keeping the physical meaning. Let us also mention that $v$ is the natural variable to prescribe the boundary data $g$.

With this new variable $v$ our initial and boundary value problem reads

$$
\begin{cases}u_{t}=\Delta v, & v=u^{m}, \\ v=g, & x \in \partial \Omega, \\ u(x, 0)=f(x), & x \in \Omega .\end{cases}
$$

Observe that the dependence on $m$ appears now only in the law relating the variables $u$ and $v$,

$$
v=\phi_{m}(u), \quad \phi_{m}(u)=u^{m} .
$$

Formally, if we let $m \rightarrow \infty$ we get

$$
\begin{cases}u_{t}=\Delta v, & v \in \phi_{\infty}(u), \\ v=g, & x \in \partial \Omega, \\ u(x, 0)=f(x), & x \in \Omega,\end{cases}
$$

where $\phi_{\infty}(u)$ is the monotone graph

$$
\phi_{\infty}(u)= \begin{cases}0, & 0<u<1, \\ {[0, \infty),} & u=1 \\ \emptyset, & u>1\end{cases}
$$

Problem (1.5)-(1.6) is known as the Hele-Shaw problem [10,15]. In the case $n=2$ this problem is a two-dimensional mathematical model describing the movement of a viscous incompressible fluid confined in a narrow cell between two parallel plates, [12,22]. form

A different approach to have a picture of this limit consists of writing Eq. (1.1) in the

$$
u_{t}=\nabla \cdot(D(u) \nabla u), \quad D(u)=m u^{m-1} .
$$

In the limit $m \rightarrow \infty$ the diffusivity $D(u)$ becomes infinite above the level $u=1$ and zero below it. This produces the instantaneous collapse of any piece of the solution lying above $u=1$ and tends to preclude any evolution below this level. Thus, in the absence 
of a boundary or if the boundary data are trivial, there is no evolution, as $v$ becomes zero instantaneously. However, as we shall see, the presence of non-vanishing boundary data keeps $v$ away from being zero, forcing a non-stationary Hele-Shaw flow.

To put things into perspective we will briefly summarize the results already known for the problem. Let us start with the Cauchy problem (1.1)-(1.2), $\Omega=\mathbb{R}^{n}$. In this case $\partial \Omega=\emptyset$, and no boundary data are needed. If $f$ is in the domain of $\phi_{\infty}$, i.e., if $\|f\|_{\infty} \leqslant 1$, then, by a general stability result the solutions $u_{m}$ converge to the solution $u_{\infty}$ of

$$
\begin{cases}u_{t}=\Delta v, & v \in \phi_{\infty}(u), \\ u(x, 0)=f(x), & x \in \Omega,\end{cases}
$$

see [3]. As in this case there are no boundary data, the limiting function $u_{\infty}$ is stationary. In fact $u_{\infty}(x, t)=f(x)$ for all $t>0$.

The convergence result holds true for any sequence of constitutive functions $\phi_{m}(u)$ such that $\lim \phi_{m}=\phi_{\infty}$. An important example is the Stefan problem

$$
\phi_{m}(u)=m(u-1)_{+} .
$$

In this case $u$ stands for the enthalpy and $v=\phi_{m}(u)$ represents the temperature. The specific heat is given by $c=1 / m$. Thus the Hele-Shaw problem can be seen as the zero specific heat limit of the Stefan problem $[9,16,19,23]$.

On the other hand, if the set $\{f(x)>1\}$ has positive measure, the initial data are inconsistent with the limiting graph $\phi_{\infty}(u)$, and the limit is singular. There is still convergence to a stationary profile. However, it is not $f(x)$, but the result of the projection of $f$ to a new function $u_{\infty}=u_{\infty}(x)$, such that $0 \leqslant u_{\infty} \leqslant 1$. These bounds on $u_{\infty}$ ensure that the limit profile is compatible with the limiting graph (1.6). To be more precise, it is proved in [8] that $u$ converges to $u_{\infty}$ in $L^{\infty}\left(\mathbb{R}^{n}\right)$ in the weak star topology, with

$$
u_{\infty}=f+\Delta w_{\infty}
$$

where $w_{\infty}$ is the solution to the complementarity problem

$$
(1-f-\Delta w) w=0, \quad w \geqslant 0, \quad 1-f-\Delta w \geqslant 0
$$

in $\mathbb{R}^{n}$. A proof for the cases $n=1$ and $n=2$, with $f$ radially symmetric, was independently given in [21]. A heuristic derivation was given in [11]. The result also holds for general nonlinearities $\phi_{m}(u) \rightarrow \phi_{\infty}(u)$, see [14].

For $\Omega$ bounded there are also some results in the case of homogeneous Dirichlet boundary data, $g \equiv 0$. These results are given in [4] if the initial data are consistent. For inconsistent initial data we have the paper [2] where the authors prove that $u_{m}$ converges to $u_{\infty}$ as in (1.7), with $w$ satisfying (1.8) in $\Omega$ together with the boundary condition $w=0$ for $x \in \partial \Omega$. Once more, the limit profile is stationary. In that paper the authors also improve the convergence for the case of the Cauchy problem showing convergence of $u_{m}(\cdot, t)$ in $L^{1}(\Omega)$, uniformly for $t$ in compact subsets of $(0, \infty)$.

What about nontrivial boundary data? If the initial data are consistent, then it was proved in [15] that the solutions $u_{m}$ converge to a solution of (1.5) with boundary data 
$v=g$ and initial data $u(\cdot, 0)=f$. The limit function $u_{\infty}(x, t)$ is no longer stationary and the estimates required for the proof become more involved.

There is still a gap in the theory: the case of nontrivial boundary data with inconsistent initial data. Our first result covers this gap for the case of stationary boundary data $g=g(x)$.

THEOREM 1.1. - For $f$ measurable, bounded and compactly supported let us consider the projection of $f$ into a mesa, namely

$$
\tilde{f}(x)=f+\Delta w,
$$

where $w$ satisfies the complementarity problem

$$
(1-f-\Delta w) w=0, \quad w \geqslant 0, \quad 1-f-\Delta w \geqslant 0, \quad x \in \Omega,
$$

and the boundary condition

$$
w=0, \quad x \in \partial \Omega \text {. }
$$

Let $g$ be the trace of a function in $W^{1,2}(\Omega) \cap L^{\infty}(\Omega)$ and $\left(u_{\infty}, v_{\infty}\right)$ be the solution of the Hele-Shaw problem (1.5) with initial data $u_{\infty}(x, 0)=\tilde{f}(x)$ and boundary data $v_{\infty}(x, t)=g(x)$ for $x \in \partial \Omega$. Then, as $m \rightarrow \infty$ we have

$$
\begin{array}{lll}
u_{m}(\cdot, t) \rightarrow u_{\infty}(\cdot, t) & \text { in } L^{1}(\Omega), & \text { for all } t>0, \\
v_{m} \rightarrow v_{\infty} & \text { in } L^{1}\left(\Omega \times\left(T_{1}, T_{2}\right)\right), & \text { for all } T_{2}>T_{1}>0 .
\end{array}
$$

The limit is, also in this case, non-stationary. There is a projection of the initial data $f$ to $\tilde{f}$, followed by an evolution according to Hele-Shaw. The boundary data produce the evolution. In fact, when the boundary data vanish the limit is stationary, and we only have the projection onto a 'mesa'. Note that the boundary data play no role in the projection of the initial data.

The non-stationary character of the limit solution introduces some technical difficulties that are not present in the case with vanishing boundary data. Indeed, for $t=0$ the functions $v_{m}$ become enormous for $m$ large. Thus, it is impossible to obtain uniform bounds down to $t=0$. However, if the limit is stationary, one can avoid this problem just by studying convergence away from $t=0$, say $t=1$, and then transport this information to other times in a suitable way. This idea does not work in the case of a non-stationary limit, and we need to analyze first this singularity, by investigating the boundary layer that appears for very small times, in the very fast transition between the initial data $f$ and its projection $\tilde{f}$.

Next we discuss the formation of this boundary layer. In order to be able to see the collapse of the initial data, we have to change the time scale. For the Cauchy problem, $\Omega=\mathbb{R}^{n}$, this task was performed in [13]. The authors consider the function

$$
\bar{u}_{m}(x, t):=u_{m}(x, \tau(t)), \quad \tau(t)=t^{m} .
$$


The new time scale is suggested by the asymptotic behaviour of the Barenblatt solution, see formula (1.16) below. They prove that $\bar{u}_{m} \rightarrow \bar{u}_{\infty}$ weakly star in $L_{\text {loc }}^{\infty}\left(\mathbb{R}^{n} \times(0, \infty)\right)$ as $m \rightarrow \infty$, where, for a.e. $t$,

$$
\bar{u}_{\infty}(\cdot, t)=f+\Delta \bar{w}_{\infty},
$$

$\bar{w}_{\infty}(\cdot, t)$ being the solution of the variational inequality

$$
(1 / t-f-\Delta w) w=0, \quad w \geqslant 0, \quad 1 / t-f-\Delta w \geqslant 0
$$

in $\mathbb{R}^{n}$. Thus, in the new time scale we have a projection into a mesa of height $1 / t$. A similar result for general approximating nonlinearities $\phi_{m} \rightarrow \phi_{\infty}$ is given in [14].

Our second theorem is the counterpart of the results in [13] for the case in which the boundary $\partial \Omega$ is not empty. For $m \in(1, \infty)$ we introduce

$$
\bar{u}_{m}(x, t)=u_{m}(x, \tau(t)), \quad \tau(t)=\frac{t^{m+1}}{m+1} .
$$

THEOREM 1.2. - Let $\bar{w}_{\infty}(\cdot, t)$ be the solution to the complementarity problem

$$
(1 / t-f-\Delta w) w=0, \quad w \geqslant 0, \quad 1 / t-f-\Delta w \geqslant 0, \quad x \in \Omega,
$$

with boundary data

$$
w(x, t)=0, \quad x \in \partial \Omega,
$$

and let

$$
\bar{u}_{\infty}(\cdot, t)=f+\Delta \bar{w}_{\infty}(\cdot, t) .
$$

Then, for all $0<t \leqslant 1$,

$$
\bar{u}_{m}(\cdot, t) \rightarrow \bar{u}_{\infty}(\cdot, t) \quad \text { in } L^{1}(\Omega) \text { as } m \rightarrow \infty .
$$

Note that the boundary layer connects the states $f$ and $\tilde{f}$, since the final stage $\bar{u}_{\infty}(\cdot, 1)$ is precisely the projected initial data $\tilde{f}$. The boundary data $g$ play no role in the description of the projection, which may have been expected since $f$ and $\tilde{f}$ are independent of $g$.

Our proof of Theorem 1.2 also applies to the Cauchy problem. Thus we improve the result in [13], as we have better convergence.

We point out that the whole limiting evolution, i.e., the very fast projection followed by the Hele-Shaw flow, can be described in an unified way by means of a variational inequality formulation, see Section 3 for details. This formulation consists of the complementarity problem (1.9) together with the boundary condition

$$
w(x, t)=g(x) t, \quad x \in \partial \Omega .
$$

The solution $\left(u_{\infty}, v_{\infty}\right)$ for the Hele-Shaw problem (1.5) can be computed from $w$ through the formulas

$$
u_{\infty}=f+\Delta w, \quad v_{\infty}=w_{t} .
$$


Note that also in Theorem 1.2 we use a variational inequality formulation to characterize the evolution in the boundary layer time scale. It is worth mentioning that these variational formulations are quite convenient from the computational point of view, as the time $t$ enters only as a parameter.

Theorem 1.1 proves the convergence of the variables $u_{m}$ and $v_{m}$ to their counterparts in a Hele-Shaw evolution. However, it says very little about the positivity sets of solutions of the PME for $m$ large. Our next purpose is to investigate the behaviour of these positivity sets in the limit $m \rightarrow \infty$. The above mentioned variational inequality formulation (1.9), (1.14), (1.15) will be rather useful in this task.

Since $v_{m}$ is a power of $u_{m}$, both functions share the same support for finite $m$. We will prove that the limit of the positivity sets of $u_{m}(\cdot, t)$ and $v_{m}(\cdot, t)$ is a set that can be regarded as the positivity set of $u_{\infty}(\cdot, t)$. However, this set does not coincide in general with the positivity set of $v_{\infty}(\cdot, t)$. Though $u_{\infty}$ cannot vanish in sets where $v_{\infty}$ is positive, as $v_{\infty} \in \phi_{\infty}\left(u_{\infty}\right)$, the opposite case is possible, and there are indeed many examples of Hele-Shaw flows such that $v_{\infty}$ vanishes in sets where $u_{\infty}$ is positive.

To state our result precisely we define $S_{\infty}(t)$ for fixed $t$ as

$$
S_{\infty}(t)=\{f>0\} \cup\{w(\cdot, t)>0\},
$$

where $w$ is the solution to the complementarity problem (1.9), (1.14). The set $S_{\infty}(t)$ may be thought as the positivity set for $u_{\infty}(\cdot, t)$, see (1.9) and (1.15).

THEOREM 1.3. - For each positive $t$ we have

$$
\lim _{m \rightarrow \infty}\left\{u_{m}(\cdot, t)>0\right\}=S_{\infty}(t)
$$

in the sense of convergence with respect to the Hausdorff distance.

We also investigate the behaviour of the positivity sets in the boundary layer time scale. In fact we will need the description of this behaviour in order to proceed for larger times and give a proof of Theorem 1.3. We state our result in terms of the support of $\bar{u}_{m}$ (which coincides with the support of $\bar{v}_{m}$ ), and of

$$
\bar{S}_{\infty}(t)=\{f>0\} \cup\{\bar{w}(\cdot, t)>0\},
$$

where $\bar{w}$ is the function giving the evolution in the boundary layer, which is completely determined by (1.11)-(1.12). Observe that we may think of $\bar{S}_{\infty}(t)$ as the positivity set of $\bar{u}_{\infty}(\cdot, t)$, see $(1.11)$ and $(1.13)$.

THEOREM 1.4. - For $t \in[0,1]$ we have

$$
\lim _{m \rightarrow \infty}\left\{\bar{u}_{m}(\cdot, t)>0\right\}=\bar{S}_{\infty}(t)
$$

in the sense of convergence with respect to the Hausdorff distance.

Regarding the proofs, let us mention that once the right formulations for the limit problems are at hand, the main task to pass to the limit is getting enough estimates to ensure compactness. When obtaining the required bounds the existence of a boundary 

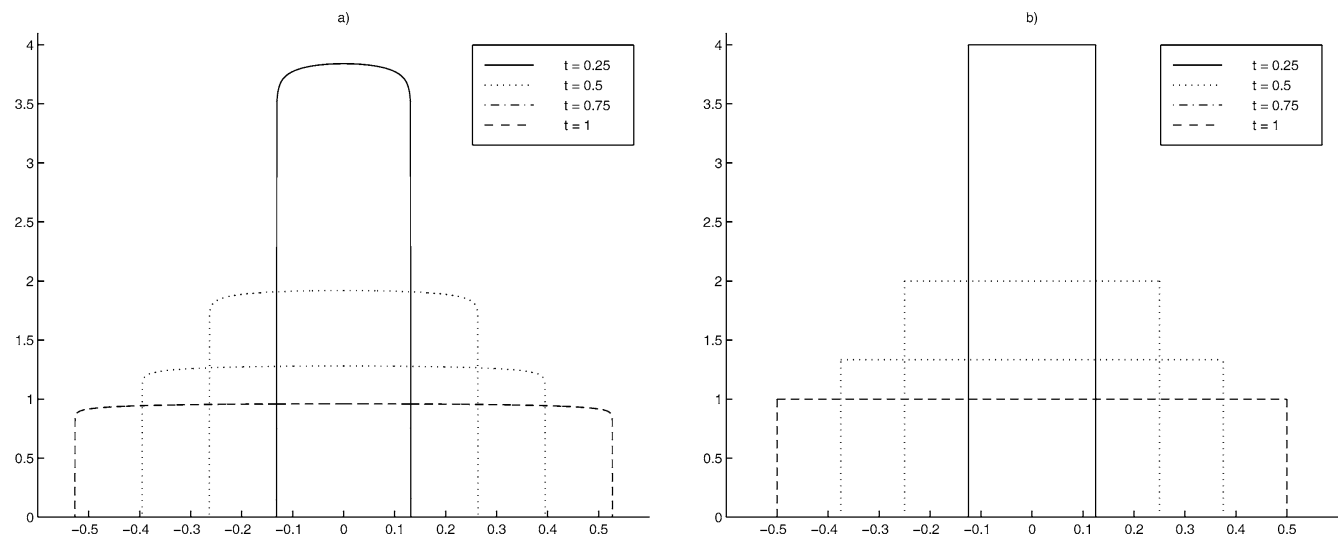

Fig. 1. $\bar{U}_{m}$ vs. $x$ for different values of $t$. (a) $m=50$. (b) $m=\infty$.

layer is clearly displayed, and the time scale that we will use to analyze the boundary layer becomes apparent. To get the estimates we will perform comparison with Barenblatt source type solutions

$$
U(x, t ; C)=t^{-\frac{n}{n(m-1)+2}}\left(C-\frac{m-1}{2 m(n(m-1)+2)}\left(\frac{|x|}{t^{\frac{1}{n(m-1)+2}}}\right)^{2}\right)_{+}^{\frac{1}{m-1}},
$$

that take the measures $M \delta_{0}$ as initial data. The constant $C$ can be computed in terms of $M, m$ and the space dimension $n$.

Barenblatt solutions are an explicit example that illustrates some features of the behaviour of solutions of the PME for large $m$. However, they do no fit into the framework of our theory, since their initial data are singular measures and we are only dealing with bounded, compactly supported, measurable functions. To have a picture of the fast collapse that occurs for large $m$ and small times we take $m=50, n=1$, $M=1$ and consider the corresponding solution $U_{m}(x, t ; C)$. From $U_{m}$ we define $\bar{U}_{m}$ as in (1.10). We plot in Fig. 1(a) the function $\bar{U}_{m}$, for four different times. Note that the time scale we are using is the one suitable to stretch the boundary layer. In Fig. 1(b) we show the limit evolution for the boundary layer $(m=\infty)$. In the original time scale the limit for a Barenblatt solution is a stationary profile, that corresponds to the final stage of the evolution shown in Fig. 1(b).

The limit $m \rightarrow \infty$ for the PME in bounded domains with homogeneous Neumann boundary conditions has been treated in [4] for consistent initial data, and in [2] for inconsistent initial data. The case of non vanishing data is considered in [17]. In this last case, the limit evolution is Hele-Shaw only up to a finite time $T$, which corresponds to the time when the Hele-Shaw flow completely fills the container represented by the domain $\Omega$. A semigroup theoretical approach to these kind of problems can be found in [5], where the authors apply this machinery to the PME with an injection term and homogeneous boundary data. The limit $m \rightarrow \infty$ for the doubly nonlinear equation

$$
u_{t}=\Delta_{p} u^{m}
$$


in $\mathbb{R}^{n}$ has been considered in [6], and in [1] for the particular case of self-similar focusing solutions. Note that the PME is (1.17) with $p=2$.

To finish this introduction, let us mention that a similar phenomenon (a projection followed by an evolution) has been observed for changing sign solutions of the PME in the limit $m \downarrow 0$, see [20].

Organization of the paper. In Section 3 we give a sketch of the proof of Theorem 1.1. However, in order to prove this theorem we need to analyze previously the boundary layer describing the collapse of the initial data. This is done in Section 2, where we give a sketch of the proof of Theorem 1.2. For the reader's sake we have collected all the cumbersome, but necessary, estimates in Section 4. We first derive them in a form suitable for the analysis of the limit in the original time scale. Then, in Section 4.1 we rewrite the estimates in a way that allows to handle the limit for the boundary layer. Finally, we devote Section 5 to the proofs of the results about the convergence of the positivity sets, Theorems 1.3 and 1.4 .

\section{The limit in the boundary layer time scale}

The main goal of this section is the description of the boundary layer that occurs in the nearly instantaneous collapse of the pieces of the initial data $f$ above the level $f=1$ when $m$ is very large.

We seek a time scale $\tau$ that allows to see the very fast evolution due to the big diffusivity at the levels $u>1$. For $m \in(1, \infty)$ we introduce

$$
\bar{u}_{m}(x, t)=u_{m}(x, \tau(t)) .
$$

We have

$$
\bar{u}_{m, t}(x, t)=u_{m, t}(x, \tau(t)) \tau^{\prime}(t)=\tau^{\prime}(t) \Delta u_{m}^{m}(x, \tau(t)) .
$$

Then we choose $\tau=t^{m+1} /(m+1)$ and

$$
\bar{v}_{m}(x, t)=\left(t \bar{u}_{m}(x, t)\right)^{m}
$$

to get

$$
\bar{u}_{m, t}=\Delta \bar{v}_{m} .
$$

Note that this choice of $\tau$, and the definition of the new variables $\bar{u}$ and $\bar{v}$, formally produce an equation for $\bar{u}$ and $\bar{v}$ which does not depend on $m$.

In order to characterize the limit we will introduce the Baiocchi type variable

$$
\bar{w}_{m}(x, t)=\int_{0}^{t} \bar{v}_{m}(x, s) d s .
$$

Integration of $(2.1)$ in $(0, t)$ yields

$$
\bar{u}_{m}(x, t)-f(x)=\Delta \bar{w}_{m}(x, t) .
$$


In addition, the following boundary condition holds

$$
\bar{w}_{m}(x, t)=\frac{t^{m+1}}{m+1} g(x) \quad x \in \partial \Omega .
$$

Next lemma is a consequence of the estimates obtained in Section 4.1.

LEMMA 2.1. - There exist functions $\bar{u}_{\infty}, \bar{v}_{\infty}$ and $\bar{w}_{\infty}$ such that we have:

(1) $\bar{u}_{\infty} \in L^{\infty}\left((0,1): L^{p}(\Omega)\right)$ for $1 \leqslant p \leqslant \infty$. For all $0 \leqslant t \leqslant 1$ the sequence $\bar{u}_{m}(\cdot, t)$ converges strongly in $L^{p}(\Omega)$ to $\bar{u}_{\infty}(\cdot, t)$, for $1 \leqslant p<\infty$, and $\bar{u}_{m}$ converges to $\bar{u}_{\infty}$ in $L^{1}(\Omega \times(0,1))$.

(2) $\bar{v}_{\infty} \in L^{\infty}(\Omega \times(0,1))$ and $\bar{v}_{m}$ converges to $\bar{v}_{\infty}$ in $L^{p}(\Omega \times(0,1))$ for $1 \leqslant p<\infty$.

(3) $\bar{w}_{\infty} \in L^{\infty}\left((0, T): W^{1, p}(\Omega)\right)$, for $1<p<\infty$. Moreover, for all $0 \leqslant t \leqslant 1$ the sequence $\bar{w}_{m}(\cdot, t)$ converges to $\bar{w}_{\infty}(\cdot, t)$ weakly in $W^{1, p}(\Omega)$ and strongly in $L^{p}(\Omega)$.

The functions $\bar{u}_{\infty}, \bar{v}_{\infty}$ and $\bar{w}_{\infty}$ can be completely characterized by means of a complementarity problem where $t$ appears only as a parameter.

LEMMA 2.2. - The function $\bar{w}_{\infty}$ satisfies the complementarity problem (1.11) with the boundary condition (1.12), and $\bar{u}_{\infty}$ and $\bar{w}_{\infty}$ satisfy (1.13). The function $\bar{v}_{\infty}$ is given by the time derivative, in the sense of distributions,

$$
\bar{v}_{\infty}=\bar{w}_{\infty, t} .
$$

Proof. - Just pass to the limit in (2.3) to get (1.13). The inequality $\bar{w}_{\infty} \geqslant 0$ is straightforward. Since $t \bar{u}_{m}=\bar{v}_{m}^{1 / m}$ and the $\bar{v}_{m}$ 's are uniformly bounded - see estimates in Section $4.1-$ we have $0 \leqslant \bar{u}_{\infty} \leqslant 1 / t$. This, together with (1.13) imply the last inequality in (1.11).

Since

$$
t \bar{u}_{m} \bar{v}_{m}=\bar{v}_{m}^{(m+1) / m},
$$

in the limit we obtain

$$
\left(t \bar{u}_{\infty}-1\right) \bar{v}_{\infty}=0,
$$

that is,

$$
t \bar{u}(x, t) \in H(\bar{v}(x, t)) .
$$

Note that we only need to put $\bar{w}_{\infty}$ instead of $\bar{v}_{\infty}$ in (2.4) to have the complementarity condition in (1.11). Formula (2.2) holds in the limit $m=\infty$. Moreover, the limit $\bar{v}_{\infty}$ satisfies

$$
\bar{v}_{\infty, t}(x, t) \geqslant-\frac{\bar{v}_{\infty}(x, t)}{t} .
$$

This happens because this inequality holds for the 'pressures' $v_{m}$ of solutions of the PME, and the definition of $\bar{v}_{m}$. Estimate (2.5) implies the retention property

$$
\bar{v}_{\infty}(x, t)>0 \Rightarrow \bar{v}_{\infty}(x, s)>0, \quad s \geqslant t>0 .
$$


Since (2.2) holds in the limit, we conclude that $\bar{v}_{\infty}$ vanishes at a point $(x, t)$ if and only if $\bar{w}(x, t)$ also vanishes.

The boundary condition (1.12) is immediate.

Remark 2.3. - For $t \leqslant 1 /\|f\|_{\infty}$, we have that $\bar{w}_{\infty}(x, t)=0$ is the solution to the complementarity problem (1.11)-(1.12). Hence $\bar{u}_{\infty}(x, t)=f(x)$ up to that time. Thus, there is a waiting time for the collapse to start. Note that the bigger the initial data, the faster the collapse begins.

\section{The limit in the original time scale}

To discuss the limit of $u_{m}$ and $v_{m}$ we will introduce again a Baiocchi type variable for $m \in(1, \infty)$, namely

$$
w_{m}(x, t)=\int_{0}^{t} v_{m}(x, s) d s, \quad x \in \Omega .
$$

It is straightforward to check that

$$
u_{m}(x, t)-f(x)=\Delta w_{m}(x, t), \quad(x, t) \in \Omega \times \mathbb{R}^{+} .
$$

Also, we have the boundary condition

$$
w(x, t)=g(x) t, \quad(x, t) \in \partial \Omega \times \mathbb{R}^{+} .
$$

The following convergence lemma is a consequence of the estimates of next section.

LEMMA 3.1. - There exist functions $u_{\infty}, v_{\infty}$ and $w_{\infty}$ such that, for any $T>0$ and $\tau$ in the interval $(0, T)$, we have:

(1) $u_{\infty} \in L^{\infty}\left((0, T): L^{p}(\Omega)\right)$ for $1 \leqslant p \leqslant \infty$. For all $t>0$ the sequence $u_{m}(\cdot, t)$ converges strongly in $L^{p}(\Omega)$ to $u_{\infty}(\cdot, t)$, for $1 \leqslant p<\infty$, and $u_{m}$ converges to $u_{\infty}$ in $L^{1}(\Omega \times(0, T))$.

(2) $v_{\infty} \in L^{p}(\Omega \times(0, T))$ for $1 \leqslant p \leqslant \infty$, and $v_{m}$ converges to $v_{\infty}$ in $L^{1}(\Omega \times(\tau, T))$.

(3) $w_{\infty} \in L^{\infty}\left((0, T): W^{1, p}(\Omega)\right)$, for $1<p<\infty$. Moreover, for all $t>0$ the sequence $w_{m}(\cdot, t)$ converges to $w_{\infty}(\cdot, t)$ weakly in $W^{1, p}(\Omega)$ and strongly in $L^{p}(\Omega)$.

The functions $u_{\infty}, v_{\infty}$ and $w_{\infty}$ can be characterized by means of a complementarity problem where $t$ appears only as a parameter. This is the content of our next lemma.

LEMMA 3.2. - For $t>0$ Eq. (3.2) also holds in the limit, and $v_{\infty}=w_{\infty, t}$, where $w_{\infty}$ solves the complementarity problem (1.9) with the boundary condition (1.14).

Proof. - It is completely analogous to the proof of Lemma 2.2 except for the complementarity condition, since Eq. (3.1) is no longer true in the limit. Indeed, we will show that

$$
w_{\infty}(x, t)=w_{\infty}\left(x, 0^{+}\right)+\int_{0}^{t} v_{\infty}(x, s) d s
$$


where we have defined $w_{\infty}\left(x, 0^{+}\right)=\bar{w}_{\infty}(x, 1)$. Let us write, for finite $m$,

$$
w_{m}(x, t)=\bar{w}_{m}(x, 1)+\int_{1 /(m+1)}^{t} v_{m}(x, s) d s .
$$

Since the functions $v_{m}(x, t)$ are uniformly bounded from above on $t \geqslant C / m$, with $C$ any positive constant (see estimates in Section 4, we apply the Dominated Convergence Theorem and our previous results about the boundary layer to get (3.3).

It is easy to check that $\left(1-u_{\infty}\right) v_{\infty}=0$, and that the retention property (2.6) holds for $v_{\infty}$. We will derive the complementarity condition from this facts, showing that we can put $w_{\infty}$ instead of $v_{\infty}$ in the formula. Note that there is nothing to do where $w_{\infty}=0$.

At points $(x, t)$ where $w_{\infty}$ is positive and $w_{\infty}(x, t)>w_{\infty}\left(x, 0^{+}\right)$we have $v_{\infty}(x, t)>$ 0 , because of the retention property for $v_{\infty}$ and (3.3).

We consider now a point $(x, t)$ such that

$$
0<w_{\infty}(x, t)=w_{\infty}\left(x, 0^{+}\right) .
$$

Formulas (1.11) and (1.12) imply that $w_{\infty}\left(x, 0^{+}\right)$solves the problem (1.9), plus the boundary condition (1.14) for $t=0$. Then

$$
f(x)+\Delta w_{\infty}\left(x, 0^{+}\right)=1 .
$$

Also, using the estimate

$$
u_{t} \geqslant-\frac{u}{(m-1) t}
$$

we get that the limit $u_{\infty}$ is non-decreasing with respect to $t$. Then, there exists the limit

$$
u_{\infty}\left(x, 0^{+}\right)=\lim _{t \downarrow 0} u_{\infty}(x, t) \leqslant u_{\infty}(x, t) \leqslant 1 .
$$

As a consequence of formula (3.2) with $m=\infty$, and (3.3), we have

$$
u_{\infty}\left(x, 0^{+}\right)=f(x)+\Delta w_{\infty}\left(x, 0^{+}\right) .
$$

When we combine this last equation with (3.4) we get $u_{\infty}\left(x, 0^{+}\right)=1$, and immediately $u_{\infty}(x, t)=1$, by $(3.5)$.

The boundary condition (1.14) is very easily obtained, just taking the limit $m \rightarrow \infty$ for the boundary values of $w_{m}$.

The limit $w_{\infty}(x, 0)=\lim _{m \rightarrow \infty} w_{m}(x, 0)$ vanishes but in general the function $w_{\infty}\left(x, 0^{+}\right)$does not. In fact, the latter gives a description of the result of the collapse of the initial data $f$ to $\tilde{f}(x)=u_{\infty}\left(x, 0^{+}\right)$.

After the instantaneous rearrangement of the initial mass the evolution is governed by Hele-Shaw, in a formulation that allows 'mushy regions'. This is an immediate corollary of our previous computations. 
COROLlARY 3.3. - The pair $\left(u_{\infty}, v_{\infty}\right)$ is a solution to the Hele-Shaw problem with boundary data $g$ and initial data $\tilde{f}$.

Proof. - As

$$
\tilde{f}=u_{\infty}\left(x, 0^{+}\right)=\lim _{t \downarrow 0} u_{\infty}(x, t),
$$

the assertion about the initial data is obvious. Since $v_{\infty}$ is the limit of $v_{m}$, the condition on the boundary data is also satisfied. We know that

$$
0 \leqslant u_{\infty} \leqslant 1, \quad v_{\infty} \geqslant 0, \quad\left(1-u_{\infty}\right) v_{\infty}=0
$$

Then $v_{\infty} \in \phi_{\infty}\left(u_{\infty}\right)$, where $\phi_{\infty}$ is the graph (1.6). To finish, just consider (3.2) with $m=\infty$ and (3.3), then take derivatives with respect to time to get $u_{\infty, t}=\Delta v_{\infty}$.

Remark 3.4. - We have described the whole limiting evolution in terms of the variational inequality formulation (1.9), (1.14). This contains, in a single setting, the projection of the initial data and the subsequent evolution. It is possible to formulate the Hele-Shaw problem in this variational form, which is completely equivalent to weak formulations when the initial data $f$ satisfy $0 \leqslant f \leqslant 1$ (see [15]). However, the variational formulation allows noncompatible data $f$ and produces the effect of 'projecting' them to $\tilde{f}$. A Hele-Shaw flow with initial data $\tilde{f}$ follows.

This implies the non existence of weak solutions to the Hele-Shaw problem when $\|f\|_{\infty}>1$. Let $(u, v)$ be a weak solution with initial data $f$. Then it is also a variational solution with the same initial data (see [15]). But we have just seen that $(u, v)$ should be also a weak solution with data $\tilde{f} \neq f$, and this is contradictory.

\section{Estimates}

In this section we obtain the estimates that allow to pass to the limit as $m \rightarrow \infty$ in Lemmas 3.1 and 2.1. The first step is to get some control on the sizes of the solutions $u_{m}^{m}$ and their supports by suitable comparison arguments. We will use the Barenblatt or source solutions (1.16). To get some extra flexibility for our comparison we will use this solutions shifted in time, i.e., we will consider $U(x, t+\tau ; C)$ for suitable $\tau>0$. The main idea is to choose $\tau$ and $C$ such that $U$ is greater than the initial data $f$, at $t=0$, and $U^{m}$ is greater than the boundary data $g$ on the compact set $\partial \Omega \times[0, T]$. We will notice that we cannot choose $T$ independently of the value of $m$ when doing so. However, the choice $T=1 / m$ is enough to proceed. As a second step we will handle the time interval $[1 / m, T]$, with a constant $T$.

We choose $R>0, M>1$ and $N>0$ such that

$$
f(x) \leqslant M, \quad x \in \Omega, \quad g(x) \leqslant N, \quad x \in \partial \Omega, \quad \text { supp } f \cup \partial \Omega \subset B(0, R) .
$$

Since solutions (1.16) are non-increasing with respect to the variable $|x|$, it is enough to have the inequality

$$
U(x, \tau ; C) \geqslant M, \quad|x|=R,
$$


to have a control on the initial data $f(x)=u(x, 0)$ for $x \in \Omega$. Note that this last inequality implies

$$
U^{m}(x, \tau ; C) \geqslant M^{m} \geqslant M, \quad|x|=R .
$$

If we choose $M \geqslant \max (1, N)$ we immediately get that

$$
U^{m}(x, \tau ; C) \geqslant N, \quad x \in \partial \Omega .
$$

Once we have controlled the boundary data at time $t=0$ by means of the Barenblatt solutions, it is enough to do this at time $t=T$ to get the control on the whole interval $[0, T]$. This happens because the function $U(x, \cdot ; C)$, for $x$ fixed, has only one maximum. Then, we only have to consider

$$
U^{m}(x, \tau+T ; C) \geqslant N, \quad|x|=R,
$$

together with (4.1) to perform our comparison argument.

Let us choose $\tau=\tau(m)$ and $C=C(m)$. This is a rather straightforward calculation. From (4.1) we immediately conclude

$$
C \geqslant M^{m-1} \tau^{\frac{n(m-1)}{n(m-1)+2}}+\frac{m-1}{2 m} \frac{R^{2}}{n(m-1)+2} \tau^{-\frac{2}{n(m-1)+2}} .
$$

When we take into account (4.2) we obtain

$$
C \geqslant N^{\frac{m-1}{m}}(\tau+T)^{\frac{n(m-1)}{n(m-1)+2}}+\frac{m-1}{2 m} \frac{R^{2}}{n(m-1)+2}(\tau+T)^{-\frac{2}{n(m-1)+2}} .
$$

Note that a bound for the solution $u_{m}$ is

$$
U(0, \tau ; C)=C^{\frac{1}{m-1}} \tau^{-\frac{n}{n(m-1)+2}} .
$$

Also, the support of $u(\cdot, t)$, for $t \in[0, T]$, will be contained in the ball $B(0, \bar{R})$, with

$$
\bar{R}=\left(\frac{2 m(n(m-1)+2)}{m-1} C\right)^{1 / 2}(\tau+T)^{\frac{1}{n(m-1)+2}} .
$$

So, we have to choose $C$ and $\tau$ satisfying (4.3) and (4.4) and such that (4.5) and (4.6) have bounds not depending on $m$. If we try to choose a constant $T$ then we are compelled by (4.6) to take $C \sim 1 / \mathrm{m}$. In fact, we have to do so unless we allow $T$ and $\tau$ to vanish very fast as $m \rightarrow \infty$, something we do not want to happen. The difficult point now is to get (4.3), because $M^{m}$ can be very large. We take then

$$
\tau \sim \frac{1}{m M^{m}} .
$$

It is straightforward to check that, with this choice of $\tau$, both terms in the right hand side of (4.3) are of the same order. Let us notice now that the right hand side of (4.4) has the 
same order as $N T$ as $m \rightarrow \infty$. Then, we have to choose $T=T(m) \sim 1 / m$, in order to have this inequality. Our choice for $C, \tau$ and $T$ is

$$
C=\frac{A}{m}, \quad \tau=\frac{1}{m M^{m}}, \quad T=\frac{1}{m},
$$

where $A$ is a constant depending on $M, N, R$ and $n$, but not on $m$. In the sequel we will use $A$ to denote constants of this kind, that may differ from line to line. The precise value of these constants will not be important for us.

With this choice we can control the supports and the sizes of the solutions $u_{m}$ uniformly with respect to $m$ in the time interval $[0,1 / m]$. Moreover, at time $t=1 / m$ we have

$$
u_{m}^{m}(x, 1 / m) \leqslant A,
$$

which gives an uniform control on the size of $u_{m}^{m}$ at time $T(m)$. We can then apply the arguments of [15], Section 3, to get uniform bounds on the supports of $u_{m}$ and the sizes of $u_{m}$ and $u_{m}^{m}$ on any interval $[T(m), T]$, with $T>0$ fixed. These size estimates are enough to obtain $L^{2}$ estimates for $\nabla u_{m}^{m}$ and $L^{1}$ estimates for $u_{m, t}$ and $u_{m, t}^{m}$, on any interval $\left[T_{1}, T_{2}\right]$, with $0<T_{1}<T_{2}$ fixed, see [15].

Since we want to understand, for $m \rightarrow \infty$, the limiting evolution and this is affected by the initial data $f$ we need to go down till $t=0$ with our estimates on $u_{m}$ and $u_{m}^{m}$. For $u_{m}$ we have the rather obvious bound

$$
u_{m} \leqslant M
$$

There is not an uniform bound for $u_{m}^{m}$ in $\Omega \times(0, T)$ if $\|f\|_{\infty}>1$. To deal with $u_{m}^{m}$, we will use the fact that, for our choice of $C$ and $\tau$, and $t \in[0, T(m)], u_{m}$ is below the Barenblatt $U(x, t+\tau ; C)$. Then

$$
u_{m}(x, t) \leqslant U(0, t+\tau ; C)=C^{\frac{1}{m-1}}(t+\tau)^{-\frac{n}{n(m-1)+2}}, \quad t \in[0,1 / m] .
$$

The consequences of (4.7) quoted above and (4.8) allow us to get an uniform bound for $\left\|u_{m}^{m}\right\|_{L^{1}(\Omega \times(0, T))}$ as follows. The piece in $1 / m \leqslant t \leqslant T$ is easy. Let us consider then $u_{m}^{m}$ on $\Omega \times(0,1 / m)$. We take into account the fact that the support of $u_{m}$ is contained in $B(0, \bar{R})$ and the choice $C=A / m$. Then

$$
\int_{0}^{1 / m} \int_{\Omega} u_{m}^{m}(x, t) d x \leqslant \frac{A}{m} \int_{0}^{1 / m}(t+\tau)^{-\frac{m n}{n(m-1)+2}} d t .
$$

Just use the value of $\tau=\tau(m)$ and work out the integral to get the estimate.

Now we will get the estimates to prove that $u_{m}(\cdot, t)$ converge in $L^{1}(\Omega)$ for all $t>0$. We will use Frechet-Kolmogorov's compactness criteria. The key point is that we will be able to reduce everything to the size estimate (4.8).

LEMMA 4.1. - For all $t>0$ the family $u_{m}(\cdot, t)$ is precompact in $L_{\mathrm{loc}}^{1}(\Omega)$. 
Proof. - For some suitable cut-off function $\rho$ we introduce

$$
\psi(x, t)=\rho(x)\left|u_{m}(x+h, t)-u_{m}(x, t)\right| .
$$

Then

$$
\psi_{t}(x, t)=\rho(x)\left(u_{m, t}(x+h, t)-u_{m, t}(x, t)\right) \operatorname{sign}\left(u_{m, t}(x+h, t)-u_{m, t}(x, t)\right) .
$$

Since $u_{m}$ is a solution to PME and $u \mapsto u^{m}$ is monotone

$$
\psi_{t}(x, t)=\rho(x)\left(\Delta u_{m}^{m}(x+h, t)-\Delta u_{m}^{m}(x, t)\right) \operatorname{sign}\left(u_{m}^{m}(x+h, t)-u_{m}^{m}(x, t)\right) .
$$

We apply now Kato's inequality [18]

$$
\Delta|\varphi| \geqslant \operatorname{sign} \varphi \Delta \varphi
$$

to conclude that

$$
\psi_{t}(x, t) \leqslant \rho(x) \Delta\left|u_{m}^{m}(x+h, t)-u_{m}^{m}(x, t)\right| .
$$

We integrate this inequality in $\Omega \times(0, t)$, then

$$
\int_{\Omega} \psi(x, t) d x \leqslant \int_{\Omega} \psi(x, 0) d x+\iint_{\Omega \times(0, t)} \Delta \rho(x)\left|u_{m}^{m}(x+h, t)-u_{m}^{m}(x, t)\right| d x d t .
$$

The integral in the right-hand side of this last inequality is controlled by

$$
\|\Delta \rho\|_{\infty}|h| \iint_{\Omega \times(0, t)}\left|\nabla u_{m}^{m}(x, t)\right| d x d t
$$

So, we have to estimate $\nabla u_{m}^{m}$ in $L^{1}(\Omega \times(0, t))$. In fact, we already have uniform bounds for $t \geqslant 1 / m$. Our concern now is to control the integral on $\Omega \times(0,1 / m)$. To do this we first apply Hölder's inequality with respect to the space variable to get

$$
\iint_{\Omega \times(0, t)}\left|\nabla u_{m}^{m}(x, t)\right| d x d t \leqslant|\Omega|^{1 / 2} \int_{0}^{t}\left(\int_{\Omega}\left|\nabla u_{m}^{m}(x, t)\right|^{2} d x\right)^{1 / 2} d t
$$

We look for a bound for the integral of $\left|\nabla u_{m}^{m}\right|^{2}$. We will not write the subscript $m$ in the rest of the proof. For a fixed cut-off function $\varphi(x)$, such that $0 \leqslant \varphi \leqslant 1$, we have

$$
\varphi u^{m} u_{t}=\varphi u^{m} \Delta u^{m}
$$

Also

$$
u_{t} \geqslant-\frac{u}{(m-1) t}
$$


Then

$$
-\frac{\varphi u^{m+1}}{(m-1) t} \leqslant \varphi u^{m} \Delta u^{m}
$$

We integrate in $\Omega$. Hence

$$
-\int_{\Omega} \frac{\varphi u^{m+1}}{(m-1) t} d x \leqslant \int_{\Omega}-\varphi\left|\nabla u^{m}\right|^{2} d x-\int_{\Omega} u^{m} \nabla u^{m} \nabla \varphi d x .
$$

We write

$$
u^{m} \nabla u^{m}=\frac{1}{2} \nabla u^{2 m}
$$

so that

$$
-\int_{\Omega} \frac{\varphi u^{m+1}}{(m-1) t} d x \leqslant-\int_{\Omega} \varphi\left|\nabla u^{m}\right|^{2} d x+\frac{1}{2} \int_{\Omega} u^{2 m} \Delta \varphi d x .
$$

Then, we conclude that

$$
\int_{\Omega} \varphi(x)\left|\nabla u^{m}\right|^{2}(x, t) d x \leqslant \frac{1}{(m-1) t} \int_{\Omega} \varphi(x) u^{m+1}(x, t) d x+\int_{\Omega} \Delta \varphi(x) u^{2 m}(x, t) d x .
$$

An integration with respect to time yields

$$
\begin{aligned}
& \int_{0}^{1 / m}\left(\int_{\Omega}\left|\nabla u^{m}(x, t)\right|^{2} d x\right)^{1 / 2} d t \\
& \leqslant \int_{0}^{1 / m}\left(\frac{1}{(m-1) t} \int_{\Omega} u^{m+1}(x, t) d x\right)^{1 / 2} d t+\|\Delta \varphi\|_{\infty}^{1 / 2} \int_{0}^{1 / m}\left(\int_{\Omega} u^{2 m}(x, t) d x\right)^{1 / 2} d t .
\end{aligned}
$$

Since everything is controlled for $t>1 / m$ we can use (4.9) and this last inequality to get a bound of the $L^{1}$ norm of $\nabla u^{m}$ by means of the size estimate (4.8). Computations are rather straightforward now. We recall the fact that the supports of the functions $u_{m}$ are all contained in a fixed ball with radius $\bar{R}$. This allows us to get rid of the integrals in space very easily. When we combine this fact with (4.8) we get

$$
\int_{0}^{1 / m}\left(\int_{\Omega} u^{2 m}(x, t) d x\right)^{1 / 2} d t \leqslant \frac{A}{m} \int_{0}^{1 / m}\left(t+\frac{1}{m M^{m}}\right)^{-\frac{n m}{n(m-1)+2}} .
$$

Just perform the integral in the right-hand side to check that it is uniformly bounded. We consider now

$$
\int_{0}^{1 / m}\left(\frac{1}{(m-1) t} \int_{\Omega} u^{m+1}(x, t) d x\right)^{1 / 2} d t \leqslant \frac{A}{m} \int_{0}^{1 / m} t^{-1 / 2}\left(t+\frac{1}{m M^{m}}\right)^{-\frac{n(m+1)}{2(n(m-1)+2)}} d t .
$$


We notice that $t^{-1 / 2}$ belongs to $L^{p}((0,1 / m))$ for all $p \in[1,2)$. Now we choose $q=2+1 / m$ and $p$ its conjugate exponent, and apply Hölder's inequality to estimate this last integral. Computations are straightforward.

\subsection{Estimates for the boundary layer}

Next we obtain the estimates required to pass to the limit in the boundary layer time scale. The estimates $\bar{u}_{m} \leqslant M$, supp $\bar{u}_{m}(\cdot, t) \subset B(0, R)$ and $\operatorname{supp} \bar{v}_{m}(\cdot, t) \subset B(0, R)$ for $t \in[0,1]$ are trivial. The estimate for $\bar{v}$ is obtained through comparison with the same family of Barenblatt solutions that we used before. We get

$$
\bar{v}_{m}(x, t) \leqslant t^{m} U^{m}\left(0, \frac{t^{m+1}}{m+1}+\tau ; C\right) .
$$

Thus,

$$
\bar{v}_{m}(x, t) \leqslant \frac{A}{m} t^{m}\left(\frac{t^{m+1}}{m+1}+\frac{1}{m M^{m}}\right)^{-\frac{n m}{n(m-1)+2}} .
$$

Hence,

$$
\bar{v}_{m}(x, t) \leqslant \frac{A}{t}, \quad t>\frac{1}{M},
$$

and

$$
\bar{v}_{m}(x, t) \leqslant(t M)^{m} A, \quad t<\frac{1}{M} .
$$

An immediate consequence is that $\bar{v}_{\infty}(x, t)=0$ for $t<\frac{1}{M}$.

LEMMA 4.2. - There exists a constant $C>0$, such that

$$
\left\|\nabla \bar{v}_{m}\right\|_{L^{2}(\Omega \times(0,1))} \leqslant C .
$$

Proof. - Multiply the equation $\bar{u}_{t}=\Delta \bar{v}$ by $\bar{v}-\bar{\psi}$, where $\bar{\psi}(x, t)=t^{m} \psi(x)$ and $\psi \in W^{1,2}(\Omega)$, has compact support and $\psi(x)=g(x)$ for $x \in \partial \Omega$. Then integrate in $\Omega \times(0,1)$. To conclude, use the uniform in $m$ size estimates for $\bar{u}$ and $\bar{v}$.

LEMMA 4.3. - For each $t \in[0,1]$ the sequence $\bar{u}_{m}(\cdot, t)$ is precompact in $L^{1}(\Omega)$.

Proof. - In order to apply Frechet-Kolmogorov's criteria we have to estimate, locally in $L^{1}$, the difference $\left|\bar{u}_{m}(x+h, t)-\bar{u}_{m}(x, t)\right|$ for small $h$ and $x \in \Omega_{1}$, with $\Omega_{1} \Subset \Omega$. We consider the time derivative

$$
\begin{aligned}
& \frac{d}{d t}\left|\bar{u}_{m}(x+h, t)-\bar{u}_{m}(x, t)\right| \\
& \quad=\operatorname{sign}\left(\bar{u}_{m}(x+h, t)-\bar{u}_{m}(x, t)\right)\left(\bar{u}_{m, t}(x+h, t)-\bar{u}_{m, t}(x, t)\right)
\end{aligned}
$$

to do so. Since

$$
\operatorname{sign}\left(\bar{u}_{m}(x+h, t)-\bar{u}_{m}(x, t)\right)=\operatorname{sign}\left(\bar{v}_{m}(x+h, t)-\bar{v}_{m}(x, t)\right)
$$


we get

$$
\begin{aligned}
& \frac{d}{d t}\left|\bar{u}_{m}(x+h, t)-\bar{u}_{m}(x, t)\right| \\
& \quad=\operatorname{sign}\left(\bar{v}_{m}(x+h, t)-\bar{v}_{m}(x, t)\right)\left(\Delta \bar{v}_{m}(x+h, t)-\Delta \bar{v}_{m}(x, t)\right) .
\end{aligned}
$$

We apply again Kato's inequality to get

$$
\frac{d}{d t}\left|\bar{u}_{m}(x+h, t)-\bar{u}_{m}(x, t)\right| \leqslant \Delta\left(\left|\bar{u}_{m}(x+h, t)-\bar{u}_{m}(x, t)\right|\right) .
$$

We take a cut-off function $\rho$, with compact support in $\Omega_{2} \Subset \Omega$ and such that $\left.\rho\right|_{\Omega_{1}} \equiv 1$. We multiply our last inequality by the non-negative cut-off function $\rho$ to get, after integration in time and space

$$
\begin{aligned}
& \int_{\Omega_{2}} \rho(x, t)\left|\bar{u}_{m}(x+h, t)-\bar{u}_{m}(x, t)\right| d x \\
& \leqslant \int_{\Omega_{2}} \rho(x, t)|f(x+h)-f(x)| d x \\
& \quad+\iint_{\Omega_{2} \times(0, t)} \Delta \rho(x, t)\left(\left|\bar{u}_{m}(x+h, t)-\bar{u}_{m}(x, t)\right|\right) d x d t .
\end{aligned}
$$

We estimate the right-hand side by

$$
\int_{\Omega_{2}} \rho(x, t)\left|\bar{u}_{m}(x+h, 0)-\bar{u}_{m}(x, 0)\right| d x+\|\Delta \rho\|_{L^{\infty}}|h| \iint_{\Omega_{2} \times(0, t)}\left|\nabla \bar{u}_{m}(x, t)\right| d x d t .
$$

This produces the required local $L^{1}$ estimate.

\section{Convergence of the positivity sets}

If $A$ is a subset of $\Omega$ we denote the $\delta$-neighbourhood of $A$ in $\Omega$ by $V_{\delta}(A)$, i.e.,

$$
V_{\delta}(A)=\{x \in \Omega, d(x, A)<\delta\} .
$$

We recall that $S_{\infty}(t):=\{f>0\} \cup\{w(\cdot, t)>0\}$ where $w$ is a solution to the complementarity problem (1.9), (1.14). From Section 4 we have that, for $t$ in a bounded interval of $[0, \infty)$ the sets $\left\{u_{m}(\cdot, t)\right\}$ and $S_{\infty}(t)$ have an uniform bound. We will begin by proving the easy part of Theorem 1.3, this is, that for given positive $\delta$

$$
S_{\infty}(t) \subset V_{\delta}\left(\left\{u_{m}(\cdot, t)>0\right\}\right)
$$

for all $m$ large enough. Let us consider $x \in S_{\infty}(t)$. If $f(x)>0$ then $u_{m}(x, t)$ is positive for all $m$, and $x \in\left\{u_{m}(\cdot, t)>0\right\}$, because of the retention property for the PME. If $f$ vanishes at $x$, then $w(x, t)>0$. Since $w$ is continuous, the set $A(t)=\{w(\cdot, t)>0\}$ is 
open, and $u_{\infty} \equiv 1$ on $A(t)$, because of the variational formulation (1.9) for the limit, and formula (1.15). Also, $A(t)$ is bounded and we can cover it with a finite family of balls $B_{i}=B\left(x_{i}, \delta / 2\right)$, with each $x_{i} \in A$. Note that all the intersections $B_{i} \cap A$ are non void open sets, hence

$$
\min _{i}\left\|u_{\infty}(\cdot, t)\right\|_{L^{1}\left(B_{i}\right)}=\eta>0
$$

If we take $m$ such that

$$
\left\|u_{m}(\cdot, t)-u_{\infty}(\cdot, t)\right\|_{L^{1}(\Omega)}<\eta,
$$

then $u_{m}$ cannot vanish identically in any of the balls $B_{i}$ and, as an immediate consequence,

$$
B_{i} \subset V_{\delta}\left(\left\{u_{m}(\cdot, t)>0\right\}\right) \text { for all } i \quad \Rightarrow \quad A(t) \subset V_{\delta}\left(\left\{u_{m}(\cdot, t)\right\}\right) .
$$

The proof is very similar for the analogue in Theorem 1.4, and we skip the details.

The 'difficult' part of the proofs is based in the following idea (cf. [7]), that we express by means of the physical picture for the PME that we have discussed in the introduction: if the fluid reaches the ball $B_{R / 2}$ at time $t^{0}+\sigma$, and there was no fluid in $B_{R}\left(x^{0}\right)$ at time $t^{0}$, then the pressure was big at some point of the boundary of $B_{R}\left(x^{0}\right)$ at a certain time contained in the time interval $\left(t^{0}, t^{0}+\sigma\right)$. This fact can be proved by comparison of the pressure $p$ with a suitably chosen supersolution. Such supersolution is given in the following lemma.

LEMMA 5.1. - The radial function

$$
V(x, t)=\left(C-\frac{\left(r-r_{0}\right)^{2}}{4 n t}\right)_{+}, \quad r=|x|
$$

is a supersolution of the pressure Eq. (1.4) in $\mathbb{R}^{n}$ for

$$
0<t<\frac{r_{0}^{2}}{4 n^{2} C}
$$

Proof. - Straightforward, just work out the computations and use Eq. (1.4) for the pressure.

We need to control uniformly in $m$ the movement of the fluid towards the center of a ball which is empty at a certain time. According to our previous remarks, we just need a uniform control of the size of the pressure at the boundary of the ball. We will obtain this control thanks to the uniform convergence of $v_{m}$ and $\bar{v}_{m}$ to 0 on suitable subsets that are contained in the complement of the supports of $u_{\infty}$ and $\bar{u}_{\infty}$.

LEMMA 5.2. - Given $t>0$ and $\delta>0$, we consider any $x \in \Omega$ such that $B(x, 2 \delta)$ is contained in the interior of the complement of $S_{\infty}(\cdot, t)$. Then, for given $\varepsilon>0$ we can find $M>1$ such that

$$
v_{m}(y, s)<\varepsilon, \quad \text { for all }(y, s) \in B(x, \delta) \times[1 / m, t], \quad \text { for all } m \geqslant M .
$$


Proof. - Let $h_{m}=h_{m}(y, s)$ be the solution to the problem

$$
\begin{array}{ll}
\Delta h_{m}(y)=u_{m}(y, s), & y \in B(x, 2 \delta), \\
h_{m}(y)=0, & |y-x|=2 \delta .
\end{array}
$$

Since

$$
\Delta v_{m}=u_{m, t} \geqslant-\frac{u_{m}}{(m-1) t},
$$

for fixed $s$ the function

$$
v_{m}(y, s)+\frac{h_{m}(y, s)}{(m-1) s}
$$

is subharmonic. Then, for $y \in B(x, \delta)$,

$$
v_{m}(y, s) \leqslant \frac{1}{\omega_{n} \delta^{n}} \int_{B(y, \delta)} v_{m}(z, s) d z+\frac{1}{\omega_{n} \delta^{n}} \int_{B(y, \delta)} \frac{h_{m}(z, s)}{(m-1) s} d z-\frac{h_{m}(y, s)}{(m-1) s} .
$$

In order to control the right-hand side of this inequality, we first estimate the size of $u_{m}(\cdot, s)$, for $s \in(1 / m, t)$. To do so we consider an integrated version of the inequality

$$
u_{m, s} \geqslant-\frac{u_{m}}{(m-1) s}
$$

For $s_{1}<s_{2}$ we obtain

$$
u_{m}\left(\cdot, s_{1}\right) \leqslant\left(\frac{s_{2}}{s_{1}}\right)^{1 /(m-1)} u_{m}\left(\cdot, s_{2}\right)
$$

This gives immediately

$$
u_{m}(\cdot, s) \leqslant(m t)^{1 /(m-1)} u_{m}(\cdot, t), \quad s \in[1 / m, t] .
$$

Then, applying standard theory for the Laplace equation we get that the norm of $h_{m}(\cdot, s)$ in $L^{\infty}(B(x, 2 \delta))$ is small, since $u_{m}(\cdot, t)$ converges to 0 . This bound is uniform for $s$ in $[1 / m, t]$ and gives a control on the size of the terms in the right-hand side of (5.2) where $h_{m}$ appears. We will control now the size of $v_{m}$ using (5.3). We recall from Section 4 the fact that there is a constant $M$ such that

$$
v_{m}(x, s) \leqslant M, \quad(x, s) \in \Omega \times[1 / m, t] .
$$

Then

$$
v_{m}(x, s)=v_{m}^{\frac{m-1}{m}}(x, s) u_{m}(x, s) \leqslant M^{\frac{m-1}{m}} u_{m}(x, s) .
$$

So, the integral of $v_{m}(\cdot, s)$ on the ball $B(y, \delta)$ is very small for large $m$.

We have an analogous result in the boundary layer time scale. 
LeMmA 5.3. - Given $t \in(0,1]$ and $\delta>0$, let us consider $x \in \Omega$ such that $B(x, 2 \delta)$ is contained in the interior of the complement of $\bar{S}_{\infty}(\cdot, t)$. Then $\bar{v}_{m}(x, t)$ converges uniformly to 0 in $L^{\infty}(B(x, \delta) \times[s, t])$, for any $s \in(0, t)$.

Proof. - It is based on the estimates

$$
\bar{u}_{m, t} \geqslant-\frac{m+1}{m-1} \frac{\bar{u}}{t}, \quad \bar{v}_{m, t} \geqslant-\frac{\bar{v}}{t}
$$

and is completely analogous to the proof of Lemma 5.2.

Now we have the tools we need to complete the proofs of Theorems 1.3 and 1.4. Note first that all we have to do is to show that, for given $\delta>0$, there exists a value of $M=M(\delta)$ such that for all $m \geqslant M$ we have that $u_{m}(x, t)$ (respectively $\bar{u}_{m}(x, t)$ ) vanishes at all the points $x$ that lie at a distance bigger than $\delta$ of the set $S_{\infty}(t)$ (respectively $\bar{S}_{\infty}(t)$ ). To proceed we will need size estimates on the functions $v_{m}$ and $\bar{v}_{m}$. We will get these estimates on balls contained in $\Omega$. As we did before, we can confine ourselves to consider just a finite number of balls, because we are always working with bounded sets. This observation will be important to have a control independent of $x$ on the value of $m$ such that $u_{m}(x, t)$ (or $\bar{u}_{m}(x, t)$ ) vanishes.

Step 1 . (The boundary layer for very small times.) We use the fact that $\bar{v}_{m}$ converges uniformly to 0 on the set $\Omega \times[0, T]$, for any $T<1 /\|f\|_{\infty}$, see (4.10). Also, for $t \in[0, T]$ we have $\bar{u}_{m}(\cdot, t) \rightarrow f$. Let us pick a point $(x, t)$, with $0<t<T$, such that there is a ball $B_{\delta}=B(x, \delta) \subset \mathbb{R}^{n}$ contained in the complement of $\bar{S}_{\infty}(t)$. The definition of $\bar{S}_{\infty}(t)$ implies that the initial data $f$ vanish on $B_{\delta}$. Moreover, for large $m$ the 'pressures' are arbitrarily small on the set $\partial B_{\delta} \times[0, t]$. By comparison with the supersolutions constructed in Lemma 5.1 (centered in $x$ ) we get that $u_{m}(x, t)$ vanishes on the ball $B(x, \delta / 2)$ for $m$ big enough.

Step 2. (Up to the end of the boundary layer.) We go now, in the boundary layer time scale, from a time $T \in\left(0,1 /\|f\|_{\infty}\right)$ to 1 . Note that $t=1$ corresponds to $T_{m}=1 / \mathrm{m}$ in the original time scale, and that with this choice of $T_{m}$ we are sure we have reached a time such that the fast collapse of the data has already occurred. Consider then a point $(x, t)$, with $t \in(T, 1]$, such that there is a ball $B(x, \delta)$ lying in the complement of $\bar{S}_{\infty}(t)$. This ball is also contained in the complement of $\bar{S}_{\infty}(T)$, because of the retention property for $\bar{w}$, see Section 2 . Hence, for large $m$, the ball $B(x, \delta / 2)$ completely lies outside $\left\{\bar{u}_{m}(\cdot, T)>0\right\}$. Since Lemma 5.3 ensures uniform convergence of $\bar{v}_{m}$ to 0 on the set $B(x, \delta / 2) \times[T, t]$, we can perform comparison with the supersolutions in Lemma 5.1 to get that $\bar{v}_{m}(x, \cdot)$ vanishes on $B(x, \delta / 4)$ if $m$ is large enough.

Step 3. (Beyond the boundary layer.) We come back now to the original time scale and to the variables $u_{m}$ and $v_{m}$. For $t>0$ we consider a point $(x, t)$ such that a ball $B(x, \delta)$ lies completely outside the set $S_{\infty}(t)$. For $m$ large we have that $1 / m<t$ and that $B(x, \delta) \subset\left\{S_{\infty}(1 / m)\right\}^{C}$. Lemma 5.2 ensures that $v_{m}$ is small on the boundary of $B(x, \delta / 2)$, for $s \in[1 / m, t]$. With this information we can use again a comparison argument with the supersolutions $(5.1)$ to prove that $v_{m}(\cdot, t)$ vanishes in a neighbourhood of $x$ for large $m$. 


\section{Acknowledgements}

The research of the first author has been partially funded by CSIC, Univ. de la República (Uruguay). The second author partially supported by a postdoctoral fellowship of the Spanish MEC, by the Stieltjes Institute (The Netherlands), by the TMR-network Nonlinear Parabolic Differential Equations: Methods and Applications, ERFB FMRX CT980 201, and by DGICYT project PB94-0153 (Spain). The agreement between the universities of both authors has partially supported their joint research.

We want to thank B. Gustafsson, S. Kamin and J.L. Vázquez for some stimulating discussions.

\section{REFERENCES}

[1] D.G. Aronson, O. Gil, J.L. Vázquez, Limit behaviour of focusing solutions to nonlinear diffusions, Comm. Partial Differential Equations 23 (1-2) (1998) 307-332.

[2] Ph. Bénilan, L. Boccardo, M.A. Herrero, On the limit of solutions of $u_{t}=\Delta u^{m}$ as $m \rightarrow \infty$, Rend. Sem. Mat. Univ. Politec. Torino, Fascicolo Speciale (1989) 1-13.

[3] Ph. Bénilan, M.G. Crandall, The continuous dependence on $\varphi$ of solutions of $u_{t}-\Delta \varphi(u)=$ 0, Indiana Univ. Math. J. 30 (1981) 161-177.

[4] Ph. Bénilan, M.G. Crandall, P. Sacks, Some $L^{1}$ existence and dependence results for semilinear elliptic equations under nonlinear boundary conditions, Appl. Math. Optim. 17 (3) (1988) 203-224.

[5] Ph. Bénilan, N. Igbida, Singular limit of perturbed nonlinear semigroups, Comm. Appl. Nonlinear Anal. 3 (4) (1996) 23-42.

[6] Ph. Bénilan, N. Igbida, La limite de la solution de $u_{t}=\Delta_{p} u^{m}$ lorsque $m \rightarrow \infty$, C. R. Acad. Sci. Paris Sér. I Math. 321 (1995) 1323-1328.

[7] L.A. Caffarelli, A. Friedman, Continuity of the density of a gas flow in a porous medium, Trans. Amer. Math. Soc. 252 (1979) 99-113.

[8] L.A. Caffarelli, A. Friedman, Asymptotic behaviour of solutions of $u_{t}=\Delta u^{m}$ as $m \rightarrow \infty$, Indiana Univ. Math. J. 36 (4) (1987) 711-718.

[9] A.B. Crowley, On the weak solution of moving boundary problems, J. Inst. Math. Appl. 24 (1979) 43-57.

[10] E. Di Benedetto, A. Friedman, The ill-posed Hele-Shaw model and the Stefan problem for supercooled water, Trans. Amer. Math. Soc. 282 (1) (1984) 183-204.

[11] C.M. Elliot, M.A. Herrero, J.R. King, J.R. Ockendon, The mesa problem: diffusion patterns for $u_{t}=\nabla\left(u^{m} \nabla u\right)$ as $m \rightarrow \infty$, IMA J. Appl. Math. 37 (1986) 147-154.

[12] C.M. Elliot, V. Janovský, A variational inequality approach to Hele-Shaw flow with a moving boundary, Proc. Roy. Soc. Edinburgh Sect. A 88 (1981) 93-107.

[13] A. Friedman, K. Höllig, On the mesa problem, J. Math. Anal. Appl. 123 (2) (1987) 564-571.

[14] A. Friedman, S.Y. Huang, Asymptotic behavior of solutions of $u_{t}=\Delta \phi_{m}(u)$ as $m \rightarrow$ $\infty$ with inconsistent initial values, in: Analyse Mathématique et applications, GauthierVillars, Paris, 1988, pp. 165-180.

[15] O. Gil, F. Quirós, Convergence of the porous media equation to Hele-Shaw, Nonlinear Anal. 44 (2001) 1111-1131.

[16] O. Gil, F. Quirós, J.L. Vázquez, Zero specific heat limit and large time asymptotics for the one-phase Stefan problem, Preprint, 2002.

[17] N. Igbida, The mesa-limit of the porous medium equation and the Hele-Shaw problem, Differential Integral Equations 15 (2) (2002) 129-146. 
[18] T. Kato, Schrödinger operators with singular potentials, Israel J. Math. 13 (1972) 133-148.

[19] B. Louro, J.F. Rodrigues, Remarks on the quasi-steady one phase Stefan problem, Proc. Roy. Soc. Edinburgh Sect. A 102 (1986) 263-275.

[20] A. Rodriguez, J.L. Vázquez, Obstructions to existence in fast-diffusion equations, J. Differential Equations 184 (2002) 348-385.

[21] P.E. Sacks, A singular limit problem for the porous medium equation, J. Math. Anal. Appl. 140 (2) (1989) 456-466.

[22] P.G. Saffman, G.I. Taylor, The penetration of fluid into a porous medium Hele-Shaw cell, Proc. Roy. Soc. A 245 (1958) 312-329.

[23] J.L. Vázquez, A new look at the zero specific heat limit of the Stefan problem, Preprint, 1998. 